\title{
A multiplicative analogue of complex symplectic implosion
}

\author{
Andrew Dancer ${ }^{1}$. Frances Kirwan ${ }^{2}$
}

Received: 10 February 2015 / Revised: 6 May 2015 / Accepted: 20 July 2015 /

Published online: 25 August 2015

(C) Springer International Publishing AG 2015

\begin{abstract}
We introduce a multiplicative version of complex-symplectic implosion in the case of $\operatorname{SL}(n, \mathbb{C})$. The universal multiplicative implosion for $\operatorname{SL}(n, \mathbb{C})$ is an affine variety and can be viewed as a nonreductive geometric invariant theory quotient. It carries a torus action and reductions by this action give the Steinberg fibres of $\operatorname{SL}(n, \mathbb{C})$. We also explain how the real symplectic group-valued universal implosion introduced by Hurtubise, Jeffrey and Sjamaar may be identified inside this space.
\end{abstract}

Keywords Implosion · Hyperkähler · Nonreductive quotient

Mathematics Subject Classification $\quad 53 \mathrm{C} 26 \cdot 53 \mathrm{D} 20 \cdot 14 \mathrm{~L} 24$

\section{Introduction}

In a series of papers [6-10] we investigated the notion of a universal hyperkähler implosion for a compact group $K$, by analogy with the universal symplectic implosion of [14].

We recall that the universal symplectic implosion of $K$ is a space $\left(T^{*} K\right)_{\text {impl }}$ with a Hamiltonian $K \times T$ action (where $T$ is a maximal torus of $K$ ), such that the reductions by $T$ at points in the closed positive Weyl chamber give the coadjoint orbits of $K$. These orbits are the reductions of $T^{*} K$ by the right $K$ factor in the $K \times K$ action on

Frances Kirwan

kirwan@maths.ox.ac.uk

Andrew Dancer

dancer@maths.ox.ac.uk

1 Jesus College, Turl St., Oxford OX1 3DW, UK

2 Balliol College, Broad St., Oxford OX1 3BJ, UK 
$T^{*} K$. The implosion of a general symplectic manifold $X$ with Hamiltonian $K$-action is obtained by reducing $X \times\left(T^{*} K\right)_{\text {impl }}$ by the diagonal $K$ action, producing a space $X_{\text {impl }}$ with $T$ action. The reduction of $X$ by $K$, at any element $\xi$ of a chosen positive Weyl chamber in the dual $\mathfrak{k}^{*}$ of the Lie algebra of $K$, coincides with the reduction of $X_{\text {impl }}$ by $T$ at $\xi$. In this sense the implosion abelianises the $K$ action on $X$.

In [7] we constructed an analogue of implosion for hyperkähler geometry when $K=\mathrm{SU}(n)$. As a stratified complex-symplectic space the universal hyperkähler implosion is the geometric invariant theory (GIT) quotient $\left(K_{\mathbb{C}} \times \mathfrak{n}^{0}\right) / / N$ where $N$ is a maximal unipotent subgroup of the complexified group $K_{\mathbb{C}}$, and $\mathfrak{n}^{0}$ is the annihilator in $\mathfrak{k}_{\mathbb{C}}^{*}$ of the Lie algebra $\mathfrak{n}$ of $N$. The implosion is thus the complex-symplectic quotient, in the GIT sense, of $T^{*} K_{\mathbb{C}}$ by $N$, just as the symplectic implosion is (as explained in [14]) the GIT quotient of $K_{\mathbb{C}}$ by $N$. Note that $N$ is nonreductive, so some work is needed to show that the quotient exists as an affine variety. This was shown in the case $K=\mathrm{SU}(n)$ in [7] and in general follows from results of Ginzburg-Riche [13] (see the discussion in [6]).

The universal hyperkähler implosion carries an action of $K_{\mathbb{C}} \times T_{\mathbb{C}}$ where $T$ is the standard maximal torus of $K$. The presence of non-semisimple elements in $\mathfrak{k}_{\mathbb{C}}$, and the fact that non-semisimple orbits are not closed, means that the abelianisation picture becomes more complicated than in the real symplectic case. The complex-symplectic quotients by the torus action are now the Kostant varieties; that is, the varieties in $\mathfrak{k}_{\mathbb{C}}^{*}$ obtained by fixing the values of the invariant polynomials for this Lie algebra $[4,16]$. The Kostant varieties are unions of complex coadjoint orbits. The smooth locus of a Kostant variety may be identified with the corresponding regular orbit, which is open and dense in the Kostant variety with complement of codimension at least 2.

In [7] we considered the case when $K=\mathrm{SU}(n)$. In this situation the universal hyperkähler implosion can be identified with a hyperkähler quotient using quiver diagrams, and thus can be seen to be genuinely a stratified hyperkähler space rather than just a complex-symplectic one. One may, by analogy with the symplectic case, then implode a general space with hyperkähler $\mathrm{SU}(n)$ action by taking its product with the universal implosion and performing the hyperkähler reduction by the diagonal $K$ action. For general compact groups a direct construction of a hyperkähler metric on the nonreductive quotient $\left(K_{\mathbb{C}} \times \mathfrak{n}^{0}\right) / / N$ is not yet available, although in [10] we gave an alternative approach to hyperkähler implosion via moduli spaces of solutions to Nahm's equations.

Several authors have explored multiplicative quiver diagrams, for example [3,5,22]. In this paper we consider multiplicative analogues of the quiver spaces considered in [7] and obtain an analogue of the universal hyperkähler implosion for $K=\mathrm{SU}(n)$ in the quasi-Hamiltonian setting. We obtain a moduli space of solutions to multiplicative quiver equations that may be identified with the nonreductive quotient $(\mathrm{SL}(n, \mathbb{C}) \times B) / / N$, where $B$ is the standard Borel subgroup of $\operatorname{SL}(n, \mathbb{C})$. This quotient space admits actions of $K_{\mathbb{C}}$ and of the torus $T_{\mathbb{C}}$. The reductions by the torus action give the Steinberg fibres that are the multiplicative version of the Kostant varieties. We also show how the real symplectic universal group-valued implosion of [15] may be identified with a stratified set sitting inside our complex space.

The geometric quotient $G \times{ }_{U} P$, where $G$ is a complex reductive group and $P$ is a parabolic subgroup with unipotent radical $U$, also arises in the work of Boalch [2] 
as a quasi-Hamiltonian space for the action of $G \times L$ where $L$ is the Levi factor of $P$ (see also [12] for some related ideas). In particular, if $P$ is a Borel subgroup, Boalch obtains the geometric quotient $G \times{ }_{N} B$ as a quasi-Hamiltonian $G \times T_{\mathbb{C}}$ space. If $G=\operatorname{SL}(n, \mathbb{C})$, we obtain this space as a quasi-affine variety inside the implosion $(\mathrm{SL}(n, \mathbb{C}) \times B) / / N$ (see the discussion at the end of Sect. 3 and before Definition 4.1).

Pavel Safronov has recently informed us of his work [18] which constructs versions of implosion, as stacks, for general complex semisimple groups in both the Hamiltonian and quasi-Hamiltonian settings.

\section{Hyperkähler quiver diagrams}

Let us recall the finite-dimensional approach via quiver diagrams used to construct the universal hyperkähler implosion for $K=\mathrm{SU}(n)$ in [7]. We started with the flat hyperkähler space

$$
M=M(\mathbf{n})=\bigoplus_{i=1}^{r-1} \mathbb{H}^{n_{i} n_{i+1}}=\bigoplus_{i=1}^{r-1} \operatorname{Hom}\left(\mathbb{C}^{n_{i}}, \mathbb{C}^{n_{i+1}}\right) \oplus \operatorname{Hom}\left(\mathbb{C}^{n_{i+1}}, \mathbb{C}^{n_{i}}\right)
$$

with the hyperkähler action of $U\left(n_{1}\right) \times \cdots \times U\left(n_{r}\right)$

$$
\alpha_{i} \mapsto g_{i+1} \alpha_{i} g_{i}^{-1}, \quad \beta_{i} \mapsto g_{i} \beta_{i} g_{i+1}^{-1}, \quad i=1, \ldots, r-1,
$$

with $g_{i} \in U\left(n_{i}\right)$ for $i=1, \ldots, r$. Here $\mathbf{n}$ is the dimension vector $\left(n_{1}, \ldots, n_{r}=n\right)$.

We took the hyperkähler quotient of $M(\mathbf{n})$ by the group $H=\prod_{i=1}^{r-1} \mathrm{SU}\left(n_{i}\right)$, obtaining a stratified hyperkähler space $Q=M / / / H$, with a residual action of the torus $T^{r-1}=\widetilde{H} / H$ where $\widetilde{H}=\prod_{i=1}^{r-1} U\left(n_{i}\right)$, as well as a commuting action of $\mathrm{SU}\left(n_{r}\right)=\mathrm{SU}(n)$.

The universal hyperkähler implosion for $\mathrm{SU}(n)$ was defined to be the hyperkähler quotient $Q=M / / / H$, where $M, H$ are as above with $r=n$ and $n_{j}=j$, for $j=$ $1, \ldots, n$ (i.e. the case of a full flag quiver).

As a complex-symplectic space, $Q$ (for general dimension vector) is the GIT quotient, by the complexification

$$
H_{\mathbb{C}}=\prod_{i=1}^{r-1} \operatorname{SL}\left(n_{i}, \mathbb{C}\right)
$$

of $H$, of the zero locus of the complex moment map $\mu_{\mathbb{C}}$ for the $H$ action.

The complex moment map equation $\mu_{\mathbb{C}}=0$ is equivalent to the equations

$$
\beta_{i+1} \alpha_{i+1}-\alpha_{i} \beta_{i}=\lambda_{i+1}^{\mathbb{C}} I, \quad i=0, \ldots, r-2,
$$

for (free) complex scalars $\lambda_{1}^{\mathbb{C}}, \ldots, \lambda_{r-1}^{\mathbb{C}}$. 
These equations are invariant under the action of $H_{\mathbb{C}}$ given by

$$
\begin{gathered}
\alpha_{i} \mapsto g_{i+1} \alpha_{i} g_{i}^{-1}, \quad \beta_{i} \mapsto g_{i} \beta_{i} g_{i+1}^{-1}, \quad i=1, \ldots, r-2, \\
\alpha_{r-1} \mapsto \alpha_{r-1} g_{r-1}^{-1}, \quad \beta_{r-1} \mapsto g_{r-1} \beta_{r-1},
\end{gathered}
$$

where $g_{i} \in \operatorname{SL}\left(n_{i}, \mathbb{C}\right)$.

The action of $\operatorname{SL}(n, \mathbb{C})=\operatorname{SL}\left(n_{r}, \mathbb{C}\right)$ on the quotient $Q$ is given by

$$
\alpha_{r-1} \mapsto g_{r} \alpha_{r-1}, \quad \beta_{r-1} \mapsto \beta_{r-1} g_{r}^{-1} .
$$

There is also a residual action of $\widetilde{H}_{\mathbb{C}} / H_{\mathbb{C}}$ which we can identify, in the full flag case, with the maximal torus $T_{\mathbb{C}}$ of $K_{\mathbb{C}}$. The complex numbers $\lambda_{i}$ combine to give the complex-symplectic moment map for this complex torus action.

It is often useful to consider the endomorphism

$$
X=\alpha_{r-1} \beta_{r-1} \in \operatorname{Hom}\left(\mathbb{C}^{n}, \mathbb{C}^{n}\right),
$$

which is invariant under the action of $\widetilde{H}_{\mathbb{C}}$ and transforms by conjugation under the residual $\operatorname{SL}(n, \mathbb{C})$ action.

\section{Multiplicative diagrams}

Let us now consider the multiplicative version of the quiver diagrams above. That is, we consider the quasi-Hamiltonian moment map equations for the action of $H_{\mathbb{C}}=$ $\prod_{i=1}^{r-1} \operatorname{SL}\left(n_{i}, \mathbb{C}\right)$. We refer to [1] for general background on quasi-Hamiltonian spaces. A result of Van den Bergh [20,21], shows that for length one quivers

$$
V \underset{\beta}{\stackrel{\alpha}{\rightleftarrows}} W
$$

with $1+\alpha \beta, 1+\beta \alpha$ invertible, the natural $\mathrm{GL}(V) \times \mathrm{GL}(W)$ action

$$
(\alpha, \beta) \mapsto\left(g_{2} \alpha g_{1}^{-1}, g_{1} \beta g_{2}^{-1}\right)
$$

is quasi-Hamiltonian with group-valued moment map

$$
(\alpha, \beta) \mapsto\left(1+\beta \alpha,(1+\alpha \beta)^{-1}\right) .
$$

For general quivers of the kind considered in the preceding section we have an action of $\widetilde{H}_{\mathbb{C}} \times \operatorname{GL}\left(n_{r}, \mathbb{C}\right)=\prod_{i=1}^{r} \operatorname{GL}\left(n_{i}, \mathbb{C}\right)$. We let $M_{\text {mult }}(\mathbf{n})$ denote the space of such quivers, with dimension vector $\mathbf{n}$, such that the endomorphisms $1+\alpha_{i} \beta_{i}$ and $1+\beta_{i} \alpha_{i}$ are invertible for each $i$.

We shall consider the reduced space by the action of $H_{\mathbb{C}}=\prod_{i=1}^{r-1} \operatorname{SL}\left(n_{i}, \mathbb{C}\right)$. The equations the quiver has to satisfy are now

$$
1+\beta_{i+1} \alpha_{i+1}=q_{i+1}\left(1+\alpha_{i} \beta_{i}\right)
$$


for free complex scalars $q_{i+1}, 0 \leq i \leq r-2$. (See, for example, [22] for the associated equations with $q_{i}$ fixed, that arise as moment maps for the $\widetilde{H}_{\mathbb{C}}$ action). Our invertibility conditions mean that the scalars $q_{i}$ are all nonzero.

We remark that if all $q_{i}$ equal 1 then we get the same equations $\alpha_{i} \beta_{i}=\beta_{i+1} \alpha_{i+1}$ as in the additive case with $\lambda_{i}=0$.

We now define the multiplicative analogue of the hyperkähler spaces $Q$ of Sect. 2.

Definition 3.1 Let $Q_{\text {mult }}(\mathbf{n})$ denote the GIT quotient by the reductive group $H_{\mathbb{C}}$ of the space of solutions to (1) in $M_{\text {mult }}(\mathbf{n})$.

Remark 3.2 Notice that there is a residual action of $\operatorname{GL}(n, \mathbb{C}) \times \widetilde{H}_{\mathbb{C}} / H_{\mathbb{C}}$ on $Q_{\widetilde{m}}(\mathbf{n})$. In the full flag case $\mathbf{n}=(1,2, \ldots, n)$ we may identify the complex torus $\widetilde{H}_{\mathbb{C}} / H_{\mathbb{C}}$ with the maximal torus $T_{\mathbb{C}}$ in $\operatorname{SL}(n, \mathbb{C})$.

In the full flag case $Q_{\text {mult }}(\mathrm{n})$ will be a first approximation to the multiplicative implosion. The true implosion will be a slight modification of this space, involving passing to a cover at a suitable stage of the construction.

We now collect some useful results about the multiplicative quiver equations (for general dimension vectors $\mathbf{n}$ unless otherwise stated). We first consider the endomorphism $Y=1+\alpha_{r-1} \beta_{r-1}$. This is (up to inversion) the value of the moment map for the residual $\operatorname{GL}(n, \mathbb{C})$ action and is the multiplicative analogue of the endomorphism $X=\alpha_{r-1} \beta_{r-1}$ mentioned above.

Lemma 3.3 $Y=1+\alpha_{r-1} \beta_{r-1}$ satisfies the equation

$$
(Y-1)\left(Y-q_{r-1}\right) \cdots\left(Y-q_{r-1} \ldots q_{1}\right)=0 .
$$

Proof We let $X_{k}=\alpha_{r-1} \alpha_{r-2} \ldots \alpha_{r-k} \beta_{r-k} \ldots \beta_{r-2} \beta_{r-1}$ and $X=X_{1}=\alpha_{r-1} \beta_{r-1}$. Using the equation repeatedly it is now easy to show that

$$
X_{k} X=\left(q_{r-1} \ldots q_{r-k}-1\right) X_{k}+q_{r-1} \ldots q_{r-k} X_{k+1}
$$

for $1 \leq k \leq r-1$ (interpreting $X_{r}$ as 0 ). We deduce

$$
X\left(X+1-q_{r-1}\right)\left(X+1-q_{r-1} q_{r-2}\right) \cdots\left(X+1-q_{r-1} \ldots q_{1}\right)=0
$$

which on setting $Y=1+X$ yields the result.

In the case when all $q_{i}$ are 1 , then $Y$ lies in the unipotent variety.

Remark 3.4 Let us observe that, using our equations, we have

$$
\beta_{i}\left(1+\alpha_{i} \beta_{i}-\tau\right)=q_{i}\left(1+\alpha_{i-1} \beta_{i-1}-\tau q_{i}^{-1}\right) \beta_{i}
$$

and

$$
\left(1+\alpha_{i} \beta_{i}-\tau\right) \alpha_{i}=q_{i} \alpha_{i}\left(1+\alpha_{i-1} \beta_{i-1}-\tau q_{i}^{-1}\right)
$$


It follows that $\alpha_{j}, \beta_{j}$ preserve the decomposition of the quiver into subquivers given by generalised eigenspaces. Explicitly, we have

$$
\operatorname{ker}\left(1+\alpha_{i-1} \beta_{i-1}-\tau q_{i}^{-1}\right)^{m} \underset{\beta_{i}}{\stackrel{\alpha_{i}}{\rightleftarrows}} \operatorname{ker}\left(1+\alpha_{i} \beta_{i}-\tau\right)^{m}
$$

Notice that

$$
\operatorname{ker}\left(1+\alpha_{i-1} \beta_{i-1}-\tau q_{i}^{-1}\right)^{m}=\operatorname{ker}\left(1+\beta_{i} \alpha_{i}-\tau\right)^{m}
$$

using our equations (1) and the fact that $q_{i}$ are nonzero. So the maps $\alpha_{i}, \beta_{i}$ in (2) are isomorphisms unless $\tau=1$.

Remark 3.5 As in the additive case we see that $\widetilde{H}_{\mathbb{C}}=\prod_{i=1}^{r-1} \operatorname{GL}\left(n_{i}, \mathbb{C}\right)$ acts freely on a quiver if, for each $i$, either $\alpha_{i}$ is injective or $\beta_{i}$ is surjective. If for each $i$, both conditions hold, then the quiver is stable for the $\widetilde{H}_{\mathbb{C}}$ action.

If all $\alpha_{i}$ are injective or all $\beta_{i}$ are surjective, then the quiver is stable for the $H_{\mathbb{C}}$ action.

Remark 3.6 As in [8] we can look at (full flag) quivers where $\alpha_{k}, \beta_{k}$ are of the following special 'toric' form:

$$
\alpha_{k}=\left(\begin{array}{ccccc}
v_{1}^{k} & 0 & 0 & \cdots & 0 \\
0 & v_{2}^{k} & 0 & \cdots & 0 \\
& & \cdots & & \\
0 & \cdots & 0 & 0 & v_{k}^{k} \\
0 & \cdots & 0 & 0 & 0
\end{array}\right)
$$

and

$$
\beta_{k}=\left(\begin{array}{cccccc}
\mu_{1}^{k} & 0 & 0 & 0 & \cdots & 0 \\
0 & \mu_{2}^{k} & 0 & 0 & \cdots & 0 \\
& & \cdots & & & \\
0 & \cdots & 0 & 0 & \mu_{k}^{k} & 0
\end{array}\right)
$$

for some $v_{i}^{k}, \mu_{i}^{k} \in \mathbb{C}$. Now $\alpha_{k} \beta_{k}$ and $\beta_{k} \alpha_{k}$ are diagonal for each $k$, so our quiver equations are just the diagonal components of (1). In fact they are equivalent to

$$
\mu_{j}^{i} v_{j}^{i}=q_{i} \ldots q_{j}-1
$$

Note that $Y=1+\alpha_{n-1} \beta_{n-1}$ will also be diagonal.

Let us now focus on the full flag case, so $r=n$ and $n_{i}=i$ for each $i$. If all $\beta_{i}$ are surjective, then we may use the $H_{\mathbb{C}} \times \operatorname{SL}(n, \mathbb{C})$ action to put $\beta_{i}$ in the standard form $\beta_{i}=\left(\begin{array}{ll}0 & I_{i \times i}\end{array}\right)$. We now find that $Y=1+\alpha_{n-1} \beta_{n-1}$ lies in the standard Borel of $\mathrm{GL}(n, \mathbb{C})$, with diagonal entries 


$$
1, q_{n-1}, \quad q_{n-1} q_{n-2}, \ldots, \quad q_{n-1} \ldots q_{1} .
$$

Using the equations, and the fact that $\beta_{i}$ are in standard form one may work down the quiver finding $\alpha_{i}$ successively from $Y$. Conversely, every such $Y$ arises from a solution of the equations. The invertibility condition on the endomorphisms $1+\alpha_{i} \beta_{i}$ and $1+\beta_{i} \alpha_{i}$ is equivalent to the scalars $q_{j}$ all being nonzero.

The freedom involved in putting $\beta_{i}$ in this form is the action of $N$, conjugating $Y$ and acting on $\operatorname{SL}(n, \mathbb{C})$ on the right.

Our space of quivers (with all $\beta_{i}$ surjective) satisfying the equation modulo $H_{\mathbb{C}}$ is therefore

$$
\operatorname{SL}(n, \mathbb{C}) \times{ }_{N} B_{1},
$$

where $B_{1}$ denotes the subgroup of the Borel in $\operatorname{GL}(n, \mathbb{C})$ consisting of elements with 1 as the leading term on the diagonal. The geometric quotient $\operatorname{SL}(n, \mathbb{C}) \times{ }_{N} B_{1}$ can therefore be viewed as sitting inside $Q_{\text {mult }}$ (in the full flag case) as a quasi-affine variety.

If we let $B$ denote the Borel in $\operatorname{SL}(n, \mathbb{C})$, then we have a degree $n$ cover $\rho: B \rightarrow B_{1}$ given by dividing by the leading diagonal term. More explicitly, if the diagonal entries of an element in $B$ are $z_{1}, \ldots, z_{n}$ and the diagonal entries of the corresponding element $Y$ in $B_{1}$ are $w_{1}, \ldots, w_{n}$ then

$$
w_{1}=1, \quad w_{i}=\frac{z_{i}}{z_{1}}, \quad i=2, \ldots, n, \quad z_{1}^{n}=\left(w_{2} \ldots w_{n}\right)^{-1}=(\operatorname{det} Y)^{-1}
$$

As in the additive case, we may generalise the above discussion to the case of a general quiver with dimensions $n_{1}<n_{2}<\cdots<n_{r}=n$. The space of such quivers with all $\beta$ surjective may be identified with

$$
\operatorname{SL}(n, \mathbb{C}) \times_{[P, P]} \mathcal{P}
$$

where $P$ denotes the parabolic associated to the flag with dimensions $\left(n_{1}, \ldots, n_{r}=n\right)$. Moreover $\mathcal{P}$ denotes the subvariety of $\operatorname{SL}(n, \mathbb{C})$ consisting of matrices with scalar blocks down the diagonal, of size $k_{j} \times k_{j}$ where $k_{j}=n_{j+1}-n_{j}$, and with all entries below these blocks being zero. The scalars for the blocks are those given by (5).

In the full flag case when $n_{i}=i$ for each $i$, then the parabolic $P$ is the Borel, the variety $\mathcal{P}$ is $B_{1}$, and we recover the earlier result.

Example 3.7 Let us consider the $\operatorname{SL}(2, \mathbb{C})$ case, so our quiver is just

$$
\mathbb{C} \underset{\beta}{\stackrel{\alpha}{\rightleftarrows}} \mathbb{C}^{2}
$$

Our invertibility conditions are just equivalent to

$$
1+a_{1} b_{1}+a_{2} b_{2} \neq 0
$$


where $\alpha=\left(\begin{array}{l}a_{1} \\ a_{2}\end{array}\right)$ and $\beta=\left(b_{1} b_{2}\right)$. As $H_{\mathbb{C}}=\mathrm{SL}(1, \mathbb{C})$ is trivial there are no moment map equations in this case, and no quotienting. So the quiver space is just the complement in $\mathbb{C}^{4}$ of the hypersurface $1+a_{1} b_{1}+a_{2} b_{2}=0$.

It is useful to consider a slight modification of the quiver equations so that we deal with the Borel $B$ in $\operatorname{SL}(n, \mathbb{C})$ rather than the group $B_{1}$. We achieve this by setting

$$
q_{i+1}=\frac{\widetilde{q}_{i+1}}{\widetilde{q}_{i}}, \quad i=0, \ldots, r-2,
$$

subject to the constraint $\widetilde{q}_{0} \ldots \widetilde{q}_{r-1}=1$. Our equations (1) now become

$$
\widetilde{q}_{i}\left(1+\beta_{i+1} \alpha_{i+1}\right)=\widetilde{q}_{i+1}\left(1+\alpha_{i} \beta_{i}\right)
$$

and recovering $\widetilde{q}_{j}$ from $q_{j}$ involves choosing an $n$th root of unity. In terms of the matrix $Y=1+\alpha_{n-1} \beta_{n-1}$ introduced above, we have $\widetilde{q}_{n-1}^{n}=\operatorname{det} Y$, so recovering our solutions from $Y$ involves a choice of an $n$th root of det $Y$, as in the above discussion of the cover $\rho: B \mapsto B_{1}$. We then obtain the geometric quotient $\operatorname{SL}(n, \mathbb{C}) \times{ }_{N} B$ as a moduli space of quivers with all $\beta_{i}$ surjective, sitting inside the full quiver moduli space as an open dense subset.

As remarked in Introduction, Boalch [2] has obtained a quasi-Hamiltonian $G \times T_{C}$ structure on $G \times{ }_{N} B$, for a general complex reductive group $G$. (In fact he more generally obtains a quasi-Hamiltonian $G \times L$ structure on the geometric quotient $G \times{ }_{U} P$, where $P$ is a parabolic subgroup with unipotent radical $U$ and Levi factor $L$ ).

\section{Nonreductive GIT quotients}

We now make contact with nonreductive GIT quotients following [11]. The quotient $X / / G$, in the sense of geometric invariant theory (GIT), of an affine variety $X$ over $\mathbb{C}$ by the action of a complex reductive group $G$ is the affine variety $\operatorname{Spec} \mathcal{O}(X)^{G}$ associated to the algebra $\mathcal{O}(X)^{G}$ of $G$-invariant regular functions on $X$. This makes sense because the algebra $\mathcal{O}(X)^{G}$ is finitely generated, since $X$ is affine and $G$ is reductive. If we want to quotient an affine variety by a nonreductive group then difficulties can arise because the algebra of invariants is not necessarily finitely generated. However if the algebra of invariants is finitely generated then we can define the GIT quotient to be the affine variety associated to this algebra, just as for reductive groups.

It is worth noting that the inclusion of $\mathcal{O}(X)^{G}$ in $\mathcal{O}(X)$ induces a natural $G$-invariant morphism from $X$ to $X / / G$. When $G$ is reductive this morphism is always surjective, and points of $X$ become identified in $X / / G$ if and only if the closures of their $G$-orbits meet in $X$. However when the group is not reductive this morphism is not necessarily surjective; indeed its image is in general not a subvariety of the GIT quotient but only a constructible subset [11].

Recall that the universal symplectic implosion for a compact group $K$ can be identified with the nonreductive GIT quotient $K_{\mathbb{C}} / / N$ of the complexified group $K_{\mathbb{C}}$ (which is a complex affine variety) by the action of its maximal unipotent subgroup $N$ [14]. 
Here the algebra of invariants $\mathcal{O}\left(K_{\mathbb{C}}\right)^{N}$ is finitely generated although $N$ is not reductive. In fact $K_{\mathbb{C}} / / N$ is the canonical affine completion of the quasi-affine variety $K_{\mathbb{C}} / N$, which embeds naturally as an open subset of $K_{\mathbb{C}} / / N$ with complement of codimension at least two. The restriction map from $\mathcal{O}\left(K_{\mathbb{C}} / / N\right)$ to $\mathcal{O}\left(K_{\mathbb{C}} / N\right)$ is thus an isomorphism, and both algebras can be identified with the algebra of $N$-invariant regular functions on $K_{\mathbb{C}}$. In terms of the moment map description of the symplectic implosion, $K_{\mathbb{C}} / N$ corresponds to the open subset determined by the interior of the positive Weyl chamber for $K$.

Recall also from [7] that in the additive case when $K=\operatorname{SU}(n)$ the universal hyperkähler implosion can be identified with the GIT quotient $\left(K_{\mathbb{C}} \times \mathfrak{n}^{\circ}\right) / / N$ by the nonreductive group $N$. (On choosing an invariant inner product, the annihilator $\mathfrak{n}^{\circ}$ may be identified with the opposite Borel subalgebra $\mathfrak{b}$ ). Just as for the action of $N$ on $K_{\mathbb{C}}$, the algebra of invariants turns out to be finitely generated, and the GIT quotient is defined to be the corresponding affine variety. As the moment map for the right $K_{\mathbb{C}}$ action on $T^{*} K_{\mathbb{C}}$ is projection onto the Lie algebra factor, the quotient $\left(K_{\mathbb{C}} \times \mathfrak{n}^{\circ}\right) / / N$ can be viewed as the complex-symplectic quotient in the GIT sense of $T^{*} K_{\mathbb{C}}$ by $N$.

A natural multiplicative version of this starts with the double $K_{\mathbb{C}} \times K_{\mathbb{C}}$ instead of the cotangent bundle $T^{*} K_{\mathbb{C}}$ [1]. We have an action of $K_{\mathbb{C}} \times K_{\mathbb{C}}$, given by

$$
(u, v) \mapsto\left(g_{L} u g_{R}^{-1}, g_{R} v g_{R}^{-1}\right)
$$

with quasi-Hamiltonian moment map

$$
\left(\mu_{L}, \mu_{R}\right):(u, v) \mapsto\left(u v u^{-1}, v^{-1}\right)
$$

By analogy with the additive case we consider the nonreductive GIT quotient

$$
\left(K_{\mathbb{C}} \times B\right) / / N,
$$

where $N$ acts on the right

$$
(u, v) \mapsto\left(u n^{-1}, n v n^{-1}\right) .
$$

If $K=\mathrm{SU}(n)$, the argument in the additive case can be adapted to the present situation to show that the nonreductive GIT quotient $\left(\operatorname{SL}(n, \mathbb{C}) \times B_{1}\right) / / N$ may be identified with the space $Q_{\text {mult }}$ of solutions to the quiver equation (1) in the full flag case, modulo (in the GIT sense) the action of $H_{\mathbb{C}}=\prod_{i=1}^{r-1} \operatorname{SL}(i, \mathbb{C})$. For one shows that the resulting quiver variety is an affine variety with coordinate ring equal to the coordinate ring $\mathcal{O}\left(\operatorname{SL}(n, \mathbb{C}) \times B_{1}\right)^{N}$ of the variety of surjective quivers $\operatorname{SL}(n, \mathbb{C}) \times{ }_{N} B_{1}$. This identification of the coordinate ring is obtained by showing that $\operatorname{SL}(n, \mathbb{C}) \times{ }_{N} B_{1}$ is an open subset of the affine variety $Q_{\text {mult }}$ with complement of codimension at least two. So $Q_{\text {mult }}=\left(\operatorname{SL}(n, \mathbb{C}) \times B_{1}\right) / / N$ may be viewed as the canonical affine completion of the geometric quotient $\operatorname{SL}(n, \mathbb{C}) \times_{N} B_{1}$.

Working instead with (6) gives the analogous result for $(\operatorname{SL}(n, \mathbb{C}) \times B) / / N$ (recall that $B$ is an $n$-fold cover of $\left.B_{1}\right)$. Our quasi-Hamiltonian reduction $(\operatorname{SL}(n, \mathbb{C}) \times B) / / N$ 
(which in general may be singular) may be thus viewed as the canonical affine completion of the smooth quasi-Hamiltonian space $\operatorname{SL}(n, \mathbb{C}) \times{ }_{N} B$ as discussed at the end of Sect. 3.

Definition 4.1 The multiplicative universal complex-symplectic implosion for $\operatorname{SL}(n, \mathbb{C})$ is $\widetilde{Q}_{\text {mult }}=(\operatorname{SL}(n, \mathbb{C}) \times B) / / N$, or equivalently the GIT quotient by $H_{\mathbb{C}}$ of the space of full flag quivers satisfying (6).

The left $\operatorname{SL}(n, \mathbb{C})$ action on $\operatorname{SL}(n, \mathbb{C}) \times \operatorname{SL}(n, \mathbb{C})$ descends to the nonreductive GIT quotient $\widetilde{Q}_{\text {mult }}=(\operatorname{SL}(n, \mathbb{C}) \times B) / / N$. We also have a residual right action of $T_{\mathbb{C}}=$ $B / N$.

The left moment map $\mu_{L}$ defined above is an $N$-invariant map $(u, v) \mapsto u v u^{-1}$ which descends to a map $\widetilde{Q}_{\text {mult }} \rightarrow \operatorname{SL}(n, \mathbb{C})$. We also have a map $\psi: \widetilde{Q}_{\text {mult }} \rightarrow T_{\mathbb{C}}$ given by projecting onto the diagonal in $B$.

If we take the level set $\psi^{-1}(1)$ and reduce by $T_{\mathbb{C}}$ we obtain the affine variety $(\mathrm{SL}(n, \mathbb{C}) \times N) / / B$. This is actually the target space of the multiplicative Springer resolution

$$
\mathrm{SL}(n, \mathbb{C}) \times_{B} N \mapsto \mathcal{U}
$$

that is, it is the unipotent variety $\mathcal{U}$. The multiplicative Springer map is just $\phi:(u, v) \mapsto$ $u v u^{-1}$. The identification of $\mathcal{U}$ with $(\operatorname{SL}(n, \mathbb{C}) \times N) / / B$ is the well-known fact that the Springer map is an affinisation map.

More generally, we can reduce via $\psi$ at a level $\lambda$ in $T_{\mathbb{C}}$. We obtain the quotient $\operatorname{SL}(n, \mathbb{C}) \times \lambda . N / / B$.

Our map gives a surjection of $\operatorname{SL}(n, \mathbb{C}) \times_{B} \lambda . N$ onto the Steinberg fibre $F_{\lambda}$ which is the variety of elements in $\operatorname{SL}(n, \mathbb{C})$ where the regular class functions take the same values as they do on the diagonal matrix with entries $\lambda$.

The Steinberg fibres are the multiplicative analogues of the Kostant varieties. We recall the following facts (see [12] or [19, Section 6], for example) that hold for general complex semisimple $K_{\mathbb{C}}$ :

(i) Each Steinberg fibre $F_{\lambda}$ is a finite union of conjugacy classes. The dimension of $F_{\lambda}$ is $\operatorname{dim} K_{\mathbb{C}}-\operatorname{rank} K_{\mathbb{C}}$.

(ii) The regular elements form a single conjugacy class which is open and dense. This class is the smooth locus of $F_{\lambda}$. Its complement in $F_{\lambda}$ has complex codimension at least 2.

(iii) The semisimple elements in $F_{\lambda}$ form a single conjugacy class, the unique closed class in $F_{\lambda}$. This class is contained in the closure of each class in $F_{\lambda}$.

The map $\phi: K_{\mathbb{C}} \times_{B} \lambda . N \rightarrow F_{\lambda}$ is a resolution of singularities and is an isomorphism over a locus in the target space whose complement has codimension at least 2 . As in the additive case, we conclude that $F_{\lambda}$ is the affinisation $\left(K_{\mathbb{C}} \times \lambda . N\right) / / B$. So the reduction of $\widetilde{Q}_{\text {mult }}=(\operatorname{SL}(n, \mathbb{C}) \times B) / / N$ at level $\lambda$ gives the Steinberg fibre.

Remark 4.2 We can ask whether the Steinberg fibre could also be viewed as the reduction of $\operatorname{SL}(n, \mathbb{C}) \times{ }_{B} N$ by $T_{\mathbb{C}}$ at level $\lambda$ in the sense of GIT, since it is the affine variety associated with the appropriate algebra of invariant regular functions 
on $\operatorname{SL}(n, \mathbb{C}) \times{ }_{B} N$. However geometric invariant theory does not behave well when applied to actions on quasi-affine varieties such as $\operatorname{SL}(n, \mathbb{C}) \times{ }_{B} N$ which are not affine, since quasi-affine varieties are not determined by their algebras of regular functions even when these are finitely generated. For a reductive group action on a quasi-affine variety $X$ a categorical quotient of an open subset $X^{\mathrm{ss}}$ of $X$ is given in [17, Theorem 1.10], but this differs in general from the affine variety associated to the algebra of invariants and $X^{\text {ss }}$ does not necessarily coincide with $X$, in contrast with the case when $X$ is affine.

Remark 4.3 When $Y$ is a hyperkähler manifold with an action of $\mathrm{SU}(n)$ which is Hamiltonian in the hyperkähler sense, its hyperkähler implosion is constructed in [7] as the hyperkähler quotient of the product of $Y$ with the universal hyperkähler implosion $Q$; it has an induced action of $T$ which may be complexified with respect to any of the complex structures to an action of $T_{\mathbb{C}}$. Likewise, given a general space with quasiHamiltonian $\operatorname{SL}(n, \mathbb{C})$ action, we may take its product with $\widetilde{Q}_{\text {mult }}$ to get a space with $\operatorname{SL}(n, \mathbb{C}) \times \operatorname{SL}(n, \mathbb{C}) \times T_{\mathbb{C}}$ action, and perform fusion (cf. [1]) to obtain a space with $\operatorname{SL}(n, \mathbb{C}) \times T_{\mathbb{C}}$ action. Reducing by $\operatorname{SL}(n, \mathbb{C})$ then yields a space with $T_{\mathbb{C}}$ action.

Remark 4.4 If, for a general semisimple $K_{\mathbb{C}}$, we could show finite generation of the ring of $N$-invariants $\mathcal{O}\left(K_{\mathbb{C}} \times B\right)^{N}$, then the nonreductive GIT quotient $\left(K_{\mathbb{C}} \times B\right) / / N$ would exist as an affine variety, and the discussion of this section would go through for general $K_{\mathbb{C}}$. The analogous result in the additive case is known by work of GinzburgRiche [13], but we have not yet been able to adapt it to the multiplicative setting.

Example 4.5 Let us return to the $\operatorname{SL}(2, \mathbb{C})$ example. We are considering the quotient $(\mathrm{SL}(2, \mathbb{C}) \times B) / / N$, where as usual $B$ is the standard Borel and $N$ the associated maximal unipotent.

Let us write the elements of $\operatorname{SL}(2, \mathbb{C})$ and $B$ as $\left(\begin{array}{ll}a & b \\ c & d\end{array}\right)$ and $\left(\begin{array}{ll}e & f \\ 0 & e^{\prime}\end{array}\right)$ with relations

$$
a d-b c=1, \quad e e^{\prime}=1 .
$$

The action of $\left(\begin{array}{ll}1 & n \\ 0 & 1\end{array}\right)$ leaves $a, c, e$ and $e^{\prime}$ invariant and transforms $b, d, f$ as follows:

$$
b \mapsto b-a n, \quad d \mapsto d-c n, \quad f \mapsto f+n\left(e^{\prime}-e\right) .
$$

The invariants are generated by $a, c, e, e^{\prime}$ and

$$
x=a f+\left(e^{\prime}-e\right) b, \quad y=c f+\left(e^{\prime}-e\right) d
$$

with relations

$$
c x-a y=e-e^{\prime}, \quad e e^{\prime}=1 .
$$

In terms of new variables $X=e x$ and $Y=e y$ we can rewrite this as

$$
c X-a Y=e^{2}-1, \quad e \neq 0,
$$


that is, an open set in the complex quadric in $\mathbb{C}^{5}$. Note this can also be written as the double cover of the complement in $\mathbb{C}^{4}$ of the locus $c X-a Y=-1$, which is compatible with the quiver picture as in Example 3.7.

Remark 4.6 If we take the element of $B$ to be in the real maximal torus, that is we take $e=\exp (i \theta), e^{\prime}=\exp (-i \theta), f=0$, then the relation becomes

$$
c x-a y=2 i \sin \theta \text {. }
$$

If we take, for example, $c=i \bar{x}, a=-i \bar{y}$, then we get a copy of the group-valued symplectic implosion $S^{4}$ inside our variety. We have a copy of $S^{3}$ for each $\theta \in(0, \pi)$ and these collapse to a point at the endpoints $\theta=0, \pi$.

We can generalise this idea to produce a copy of the quasi-Hamiltonian symplectic implosion inside our complex space.

Let us take $q_{i}$ to lie in the unit circle. We choose a branch of the square root function on the half plane $\operatorname{Re} z<0$, and consider quivers of the toric form (3)-(4) where the entries are given by

$$
v_{j}^{i}=\mu_{j}^{i}=\sqrt{q_{i} \ldots q_{j}-1} .
$$

For such quivers we recall that $Y=1+\alpha_{n-1} \beta_{n-1}$ is diagonal with entries given by (5). Setting a consecutive run of entries of $Y$ to be equal is equivalent to setting a consecutive run of $q_{j}$ to be 1 . If, say, $q_{i}=\cdots=q_{i+m}=1$ for some $m \geq 0$ (so that $m+2$ consecutive entries of $Y$ are equal), then the last diagonal entry of $\beta_{i}$, the last two of $\beta_{i+1}$, and so on up to the last $m+1$ of $\beta_{i+m}$, are zero, and similarly for the corresponding $\alpha$. This means that the quiver decomposes according to the direct sum $\operatorname{ker} \alpha_{j} \oplus \operatorname{im} \beta_{j}$, where ker $\alpha_{j}=\operatorname{ker} \beta_{j-1}$ and $\operatorname{im} \beta_{j}=\operatorname{im} \alpha_{j-1}$. Moreover the quiver maps are zero on ker $\alpha_{j}$ and injective and surjective on the complement.

Let us now consider the sweep of such quivers under the action of $K=\operatorname{SU}(n)$. Now the action of $K \times H_{\mathbb{C}}=\mathrm{SU}(n) \times \prod_{i=1}^{n-1} \mathrm{SL}(i, \mathbb{C})$ preserves the scalars $q_{i}$, hence if $K$ moves a quiver to another quiver of the same form, then the two quivers must be the same modulo the action of $H_{\mathbb{C}}$. That is, $g \in \mathrm{SU}(n)$ has the same effect on the quiver as $\left(h_{2}, \ldots, h_{n-1}\right) \in H_{\mathbb{C}}$. The resulting equations

$$
\alpha_{j}=h_{j+1} \alpha_{j} h_{j}^{-1}, \quad 2 \leq j \leq n-2, \quad \alpha_{1}=h_{2} \alpha_{1}, \quad \alpha_{n-1}=g^{-1} \alpha_{n-1} h_{n-1}^{-1},
$$

together with their analogues for $\beta$, now imply that $\left(h_{2}, \ldots, h_{n-1}\right)$ and $g$ preserve the above decomposition. We get that $g$ lies in the commutator of the parabolic associated to the dimension flag of the injective/surjective quiver. More precisely, the dimensions of the injective/surjective quiver are $1,2, \ldots, i-1, i-1, \ldots, i-1, i+m+1, \ldots, n$ and we take the parabolic associated to the strictly increasing sequence $1,2, \ldots, i-$ $1, i+m+1, \ldots, n$ obtained by collapsing the chain of equalities. But as $g$ lies in the maximal compact subgroup we find that $g$ lies in $\mathrm{SU}(m+2)$, diagonally embedded in $\mathrm{SU}(n)$. 
The sweep of our quivers is now $\mathrm{SU}(n) \times_{\mathrm{SU}(m+2)} y$, where $y$ denotes the set of diagonal matrices $Y$ with $Y_{i-1}=\cdots=Y_{i+m}$. This picture now generalises in the obvious way to the case of general systems of equalities between elements of $Y$.

We obtain a space stratified by sets $y$ of diagonal matrices with entries satisfying specified equalities. Each such face gives a copy of $\mathrm{SU}(n) \times \mathrm{SU}\left(n_{1}\right) \times \cdots \times \mathrm{SU}\left(n_{r}\right) y$. The open interior face $y$ where all entries are distinct just gives $\mathrm{SU}(n) \times y$.

Finally, we pass to the cover. We work on the fundamental alcove

$$
\theta_{1}-2 \pi \leq \theta_{n} \leq \theta_{n-1} \leq \cdots \leq \theta_{2} \leq \theta_{1}
$$

in the Cartan algebra $\sum_{i=1}^{n} \theta_{i}=0$, and consider the corresponding elements $\left(e^{i \theta_{1}}, \ldots, e^{i \theta_{n}}\right)$ of $\mathrm{SU}(n)$. The map $\rho$ sends this element to $\left(1, e^{i\left(\theta_{2}-\theta_{1}\right)}, \ldots, e^{i\left(\theta_{3}-\theta_{2}\right)}\right)$ in $B_{1}$. The fibres of the map are obtained by multiplying by scalar matrices in $\mathrm{SU}(n)$, but within the alcove this means only the vertices map to the same point (the identity in $B_{1}$ ).

So upstairs in the covering space we obtain a stratified space stratified by the walls of the alcove. The strata are the sets

$$
\mathrm{SU}(n) \times \mathrm{SU}\left(n_{1}\right) \times \cdots \times \mathrm{SU}\left(n_{r}\right) y
$$

discussed above. The open stratum is $\mathrm{SU}(n) \times y$, and at the other extremes the vertices just give points. We have obtained the quasi-Hamiltonian symplectic implosion introduced in [15] as a subset of our complex quasi-Hamiltonian implosion.

Example 4.7 In the case of SU(2), we take the alcove $\theta_{1}-2 \pi \leq \theta_{2} \leq \theta_{1}$ in the Cartan algebra $\theta_{1}+\theta_{2}=0$ and the associated elements $\operatorname{diag}\left(e^{i \theta_{1}}, e^{i \theta_{2}}\right)$ of the maximal torus in $\mathrm{SU}(2)$. Equivalently, we take $0 \leq \theta \leq \pi$ and $\operatorname{diag}\left(e^{i \theta}, e^{-i \theta}\right)$. The covering map to $B_{1}$ sends this to $\left(1, e^{-2 i \theta}\right)$ and is injective in the given range except that the scalar matrices $\pm I$, corresponding to $\theta=0, \pi$ both map to the identity in $B_{1}$.

We obtain the quasi-Hamiltonian symplectic implosion by taking an open stratum $\mathrm{SU}(2) \times(0, \pi)$ and then adding point strata at the endpoints, to obtain $S^{4}$ as in [15].

Acknowledgments We thank Philip Boalch, Kevin McGerty and Pavel Safronov for valuable conversations.

\section{References}

1. Alekseev, A., Malkin, A., Meinrenken, E.: Lie group valued moment maps. J. Differential Geom. 48(3), 445-495 (1998)

2. Boalch, P.P.: Riemann-Hilbert for tame complex parahoric connections. Transform. Groups 16(1), 27-50 (2011)

3. Boalch, P.: Global Weyl groups and a new theory of multiplicative quiver varieties (2013). arXiv: 1307.1033

4. Chriss, N., Ginzburg, V.: Representation Theory and Complex Geometry. Birkhäuser, Boston (1997)

5. Crawley-Boevey, W., Shaw, P.: Multiplicative preprojective algebras, middle convolution and the Deligne-Simpson problem. Adv. Math. 201(1), 180-208 (2006)

6. Dancer, A., Doran, B., Kirwan, F., Swann, A.: Symplectic and hyperkähler implosion (2014). arXiv:1412.0437 (to appear in Proceedings of the 2013 Arbeitstagung in memory of Friedrich Hirzebruch) 
7. Dancer, A., Kirwan, F., Swann, A.: Implosion for hyperkähler manifolds. Compos. Math. 149(9), $1592-1630(2013)$

8. Dancer, A., Kirwan, F., Swann, A.: Implosions and hypertoric geometry. J. Ramanujan Math. Soc. 28A, 81-122 (2013)

9. Dancer, A., Kirwan, F., Swann, A.: Twistor spaces for hyperkähler implosions. J. Differential Geom. 97(1), 37-77 (2014)

10. Dancer, A., Kirwan, F., Röser, M.: Hyperkähler implosion and Nahm's equations (2014). arXiv: 1411.0545

11. Doran, B., Kirwan, F.: Towards non-reductive geometric invariant theory. Pure Appl. Math. Q. 3(1), 61-105 (2007)

12. Evens, S., Lu, J.-H.: Poisson geometry of the Grothendieck resolution of a complex semisimple group. Mosc. Math. J. 7(4), 613-642 (2007)

13. Ginzburg, V., Riche, S.: Differential operators on $G / U$ and the affine Grassmannian. J. Inst. Math. Jussieu 14(3), 493-575 (2015)

14. Guillemin, V., Jeffrey, L., Sjamaar, R.: Symplectic implosion. Transform. Groups 7(2), 155-184 (2002)

15. Hurtubise, J., Jeffrey, L., Sjamaar, R.: Group-valued implosion and parabolic structures. Amer. J. Math. 128(1), 167-214 (2006)

16. Kostant, B.: Lie group representations on polynomial rings. Amer. J. Math. 85(3), 327-404 (1963)

17. Mumford, D., Fogarty, J., Kirwan, F.: Geometric Invariant Theory. Ergebnisse der Mathematik und ihrer Grenzgebiete, vol. 34, 3rd edn. Springer, Berlin (1994)

18. Safronov, P.: Symplectic implosion and the Grothendieck-Springer resolution (2014). arXiv:1411.2962

19. Steinberg, R.: Regular elements of semisimple algebraic groups. Inst. Hautes Études Sci. Publ. Math. 25, 49-80 (1965)

20. Van den Bergh, M.: Double Poisson algebras. Trans. Amer. Math. Soc. 360(11), 5711-5769 (2008)

21. Van den Bergh, M.: Non-commutative quasi-Hamiltonian spaces. In: Dito, G., Lu, J.-H., Maeda, Y., Weinstein, A. (eds.) Poisson Geometry in Mathematics and Physics. Contemporary Mathematics, vol. 450, pp. 273-299. American Mathematical Society, Providence (2008)

22. Yamakawa, D.: Geometry of multiplicative preprojective algebra. Int. Math. Res. Pap. IMRP, rpn008 (2008) 